\title{
On some aspects of material behavior relating microstructure and ultrasonic higher harmonic generation
}

\begin{abstract}
This article investigates some important aspects of material behavior responsible for acoustic nonlinearity. Even though the discussion is based on a specific constitutive model used for studying higher harmonic generation, the conclusions drawn are valid in a general context. Three aspects of material bchavior, namely tension-compression asymmetry, shear-normal coupling and deformation induced anisotropy are presented. The role of each in the generation of higher harmonics along with the plausible microstructural features that contribute to such behavior is discussed. First and foremost, tension-compression asymmetry is identified to cause second (even) harmonic generation in materials. Then shear-normal coupling is identified to cause generation of secondary waves of different polarity than the primary waves. In addition, deformation induced anisotropy due to the presence of residual stress/strain and its contribution to acoustic nonlincarity is qualitatively discussed. Meso-scalc modeling aspects to accurately predict the effect of microstructure on higher harmonic generation are emphasized throughout.

Keywords: Nonlinear ultrasonics; Higher harmonic generation; Microstructure characterization.
\end{abstract}

\section{Introduction}

The need to characterize the current state of a material is fundamental to structural health monitoring (SHM), the prediction of remaining useful life, and condition based maintenance of structural systems. An ability to detect and characterize material degradation in advance of the initiation of macroscale damage would have a tremendous impact on the life cycle costs of many structural systems. State of the art SHM technologies are limited to detection of macroscale damage, but nonlinear ultrasonics (Zheng et al., 2000) (Jhang, 2009) has strong potential to shift the first detection of material degradation significantly 
earlier in the life of the structural system. In this context nonlinear ultrasonics refers to the phenomenon where a monochromatic wave propagating in the material is distorted due to the material nonlinearity. The distortion causes higher harmonics to be generated at integer multiples of the primary excitation frequency, and thus the higher harmonics are sensitive to the material nonlinearity. Physically, the material nonlinearity is associated with lattice anharmonicity; i.e., it is due to the presence of non-quadratic interatomic potentials that govern the atoms in the solid (Landau and Lifshitz, 1970). The objective of this paper is to identify aspects of material behavior that are responsible for ultrasonic higher harmonic generation in materials. These aspects are most relevant when describing the meso-scale behavior of material where the effect of microstructure is prevalent. This understanding enables us to correlate higher harmonic generation with microstructure by evaluating the contribution of the latter to the pertinent aspects of material behavior to be discussed. This study also sheds light on the features that a (meso-scale) constitutive model should incorporate for it to predict harmonic generation. Below, we present a brief review of some earlier work on the theory and experimental aspects of nonlinear ultrasound for characterizing material state.

The generation of higher harmonics in metals was discovered by Breazeale and Thompson (1963) and investigated further by Hikata et al. (1965). An interesting feature of the higher harmonics is that their amplitudes increase over their propagation distance. A simple 1D nonlinear stress-strain relation $(\operatorname{Eqn}(1))$ is often employed;

$$
\sigma=E \epsilon(1+\beta \epsilon)
$$

where $\sigma$ is the stress, ' $E$ ' is the Young's modulus, $\epsilon$ is the strain and $\beta$ is a nonlinear parameter that quantifies the nonlinearity. Hikata et al. (1965) concluded that harmonic generation in the presence of bias stresses occurs due to dislocation motion as well as lattice anharmonicity. Hikata and Elbaum (1966a) further studied the dislocation dynamics on generation of second and third harmonics and Hikata and Elbaum (1966b) conducted third harmonic experiments on single crystal aluminum to confirm the theory of Hikata and Elbaum (1966a). Buck (1976) used harmonic generation to measure internal stresses produced by dislocations. Later, Cantrell and Yost (2001) modeled second harmonic generation associated with dislocation 
dipole substructures during fatigue and provided some experimental results. Their work on fatigue is extended in a series of papers by Cantrell (2004) (2006) (2009). Cash and Cai (2011) (2012) used dislocation dynamics simulations to study the effect of a bias stress on the acoustic nonlinearity parameter. They found that an orientation dependent line energy is necessary to correlate dislocation dynamics with the acoustic nonlinearity parameter. These results contradict the previously mentioned modeling efforts. Thus, while modeling progress has clearly been made there is still a strong need for improved understanding of the correlation between higher harmonic generation and microstructure.

Furthermore, phenomenological evidence exists that higher harmonic generation is sensitive to other modes of material degradation; e.g., thermal aging (Kim and Lissenden, 2009), creep (Baby et al., 2008) (Valluri et al., 2010) (Xiang et al., 2014) and radiation damage (Matlack et al., 2012). All of these modes of degradation are linked to some type of microstructure evolution, which in turn must somehow be connected to nonlinearity that results in higher harmonic generation. In this vein Johnson and coworkers (Van Den Abeele et al., 2000a) (2000b) have coined the term mesoscopic elastic materials, meaning materials that exhibit nonlinear elastic behavior associated with some microstructural feature, such as distributed microcracks or a long macrocrack. These authors emphasize the large affect that damage has on nonlinear behavior. Our immediate interest is similar, except that we seek to characterize the fairly small effects of minute changes of the material. Additionally, most research has focused on the acoustic nonlinearity parameter $\beta$, or the relative nonlinearity parameter, which may not always provide the best description of evolving material state, especially when there are multiple mechanisms in competition. Creep may be a good example, as Xiang et al. (2014) showed the nonlinearity parameter to increase and then decrease with creep strain rather than to monotonically increase.

As ultrasound is used to probe damage in the material, it is necessary to understand the essential features of higher harmonic generation in the material. A simple model can be obtained by using the conservation of linear momentum in conjunction with Eqn(1) and a linearized strain-displacement relation, which gives the $1 \mathrm{D}$ nonlinear wave equation

$$
\rho \frac{\partial^{2} u}{\partial t^{2}}=E\left(1+\beta \frac{\partial u}{\partial x}\right) \frac{\partial^{2} u}{\partial x^{2}}
$$


which, along with the boundary condition $u(0, t)=u_{0} \cos (\omega t)$, can be solved using a perturbation approach (Cantrell, 2004) to yield

$$
u(x, t)=\frac{1}{8} \beta k^{2} u_{0}^{2} x+u_{0} \cos (k x-\omega t)-\frac{1}{8} \beta k^{2} u_{0}^{2} x \cos (2 k x-2 \omega t)
$$

where $\omega$ is the frequency of the wave and $k$ denotes its wavenumber. Clearly, both the static (zero-frequency) component and the second harmonic increase linearly with propagation distance. The modal amplitude ratio, $\frac{A_{2}}{A_{1}^{2}}=\left|\frac{\beta k^{2} x}{8}\right|$, also known as the relative nonlinearity parameter and often measured in experiments is an indirect indicator of material evolution. Here, $A_{1}$ and $A_{2}$ denote the amplitude of primary and second harmonic waves respectively. Thus, it is essential to have a thorough understanding of the plausible degradation mechanisms to evaluate the material state. It should also be pointed out that while $1 \mathrm{D}$ equations suffice for bulk wave propagation, 3D equations are more appropriate for guided wave modes.

Ultrasonic waves are sensitive to the collective effects of lattice defects (e.g., dislocation monopoles, dipoles, line lengths, precipitates, persistent slip bands). They do not however exhibit sensitivity to individual lattice defects. Thus, the comprehensive understanding provided by models at the length scale of the ultrasonic wavelength should be valuable for studying higher harmonic generation in polycrystalline metals. To that end, some salient aspects of material behavior that contribute to harmonic generation in materials are discussed and the role of each in ultrasonic higher harmonic generation is presented. In addition, relevant features of microstructure that contribute to such material behavior are outlined. These aspects provide a deeper understanding and also serve as a way to quantify the meso-scale effect of microstructure on harmonic generation. By "microstructure" we refer to any of the following that are responsible for ultrasonic higher harmonic generation: crystal lattice, dislocations, precipitates, voids, micro-cracks, etc. The focus of this article is not to quantify harmonic generation from a particular "microstructure", but to identify aspects of material behavior that are responsible for higher harmonic generation. These aspects of material behavior are general and can be employed to quantify higher harmonic generation from any "microstructure" of interest. This article therefore outlines quantifiable aspects of material behavior that need to be included in models intended to correlate ultrasonic higher harmonic 
generation with the microstructure of the material. Also, it provides a framework amenable to multi-scale homogenization analysis which is indispensible for analyzing contributions of different microstructural features that span several length-scales.

The next section introduces some definitions and notation to be used in Section 3, which discusses the important aspects of material behavior that contribute to ultrasonic harmonic generation. Finally, Section 4 provides closure.

\section{Definitions and Notation}

The basic notation (Bowen, 1989) for describing the deformation of a continuum is summarized here. The position of the material particle in the reference configuration is denoted by $\mathbf{X}$ and its position in the current configuration is denoted by $\boldsymbol{x}$. The displacement of the material particle is denoted by $\mathbf{u}(\mathbf{X}, \mathrm{t})$. Bold face letters represent vectors and tensors. The deformation gradient $(\mathbf{F})$ and displacement gradient $(\mathbf{H})$ are given by

$$
\mathbf{F}=\frac{\partial \boldsymbol{x}}{\partial \mathbf{X}} \text { and } \mathbf{H}=\frac{\partial \boldsymbol{u}}{\partial \mathbf{X}}
$$

and are related by

$$
\mathbf{F}=\mathbf{I}+\mathbf{H}
$$

where $\mathbf{I}$ is the identity tensor. The Lagrangian strain $(\mathbf{E})$ is

$$
\mathbf{E}=\frac{1}{2}\left(\mathbf{H}+\mathbf{H}^{T}+\mathbf{H}^{T} \mathbf{H}\right) .
$$

Central to the focus of the article are hyperelastic materials whose constitutive response is defined using the elastic strain energy function (Landau and Lifshitz, 1970) given by

$$
W(\mathbf{E})=\frac{1}{2} \lambda(\operatorname{tr}(\mathbf{E}))^{2}+\mu t r\left(\mathbf{E}^{2}\right)+\frac{1}{3} C(\operatorname{tr}(\mathbf{E}))^{3}+B \operatorname{tr}(\mathbf{E}) \operatorname{tr}\left(\mathbf{E}^{2}\right)+\frac{1}{3} A\left(\operatorname{tr}\left(\mathbf{E}^{3}\right)\right)
$$

where $\lambda, \mu$ are Lame's constants and $A, B$ and $C$ are third order elastic constants for the material. Equation 7 is equivalent to Murnaghan's model and $\operatorname{tr}()$ denotes the trace. 
Murnaghan constants $(l, m, n)$ and Landau-Lifshitz constants $(A, B, C)$ are related by $l=$ $B+C, m=\frac{1}{2} A+B$ and $n=A$ (Destrade and Ogden, 2010). The second Piola-Kirchhoff stress tensor $\left(\mathbf{T}_{R R}\right)$ is obtained from

$$
\mathbf{T}_{R R}=\frac{\partial W(\mathbf{E})}{\partial \mathbf{E}}
$$

From $\operatorname{Eqn}(7)$ and $\operatorname{Eqn}(8)$, we obtain

$$
\mathbf{T}_{R R}(\mathbf{E})=\lambda \operatorname{tr}(\mathbf{E}) \mathbf{I}+2 \mu \mathbf{E}+A \mathbf{E}^{2}+2 B \operatorname{tr}(\mathbf{E}) \mathbf{E}+B \operatorname{tr}\left(\mathbf{E}^{2}\right) \mathbf{I}+C(\operatorname{tr}(\mathbf{E}))^{2} \mathbf{I}
$$

where the dependence on $\mathbf{E}$ is explicitly indicated. The first Piola-Kirchhoff stress $(\mathbf{S})$ is related to it by

$$
\mathbf{S}=\mathbf{F T}_{R R}
$$

We further introduce the stress associated with linearized strain $\left(\mathbf{E}^{l}\right)$

$$
\mathbf{E}^{l}=\frac{1}{2}\left(\mathbf{H}+\mathbf{H}^{\mathbf{T}}\right)
$$

as

$$
\mathbf{T}_{R R}\left(\mathbf{E}^{l}\right)=\lambda \operatorname{tr}\left(\mathbf{E}^{l}\right) \mathbf{I}+2 \mu \mathbf{E}^{l}+A \mathbf{E}^{l^{2}}+2 B \operatorname{tr}\left(\mathbf{E}^{l}\right) \mathbf{E}^{l}+B \operatorname{tr}\left(\mathbf{E}^{l^{2}}\right) \mathbf{I}+C\left(\operatorname{tr}\left(\mathbf{E}^{l}\right)\right)^{2} \mathbf{I}
$$

and stress associated with linear material behavior as

$$
\mathbf{T}_{R R}^{l}(\mathbf{E})=\lambda \operatorname{tr}(\mathbf{E}) \mathbf{I}+2 \mu \mathbf{E}
$$

Hence $\mathbf{T}_{R R}\left(\mathbf{E}^{l}\right)$ captures the contribution of material nonlinearity to the stress tensor. On the other hand, $\mathbf{T}_{R R}^{l}(\mathbf{E})$ comes from the linear elastic strain energy function (the first two terms in Eqn 7) and captures the contribution of geometric nonlinearity. This distinction is just to enhance our study, and both material and geometric nonlinearities are to be considered when using the strain energy function in $\operatorname{Eqn}(7)$. 


\section{Aspects of material behavior responsible for higher harmonic generation}

When a continuous monochromatic wave propagates in a material, its distortion results in generation of higher harmonic frequency components. Suppose first that the distortion results in an asymmetric waveform as shown in Figure 1. The distortion is obtained by multiplying the positive half-cycle of a pure sinusoid by factors of 0.8 and 0.6 . Note that only the asymmetric waveforms (0.8 and 0.6) generate even harmonics (including the zerofrequency component) that are apparent in the frequency domain. Now presume that the distortion changes the waveform shape to symmetric triangular or trapezoidal as shown in Figures 2 and 3. Only odd harmonics are generated in these cases. Evidently, even harmonic generation is associated with asymmetry of the pulse while the odd harmonic generation is associated with the distortion that does not destroy the symmetry about the zero position. This knowledge is useful in correlating harmonic generation with aspects of material nonlinearity and thus, some aspects of the material behavior which tend to produce such distortions in the displacement/stress/strain fields in the material are investigated.

As indicated in Section 1, use of ultrasound for investigating acoustic nonlinearity of metals has always focused on measuring the ' $\beta$ ' parameter. For example, Cantrell (2004) studied the contributions of dislocation monopoles and dipoles to the ' $\beta$ ' parameter. Even though the contributions of different microstructural features (dislocation monopoles and dipoles in this case) were investigated, Eqn(1) was used for the constitutive model. That is to say that the effect of microstructural change is modeled as change in ' $\beta$ ' which effectively corresponds to a change in higher order elastic constants i.e., the coefficient of $\epsilon^{2}$ in Eqn (1). But no detailed study was presented as to which microstructural changes would contribute to specific patterns in higher harmonic generation. In this section, we present some important aspects of the material behavior associated with the strain energy function $(\operatorname{Eqn}(7))$ that provide a deeper understanding of higher harmonic generation and its relation to microstructure.

To emphasize the above point, we slightly modify the notion of higher order elastic constants as they capture only the nonlinearity of the material and are more relevant to single crystals. Suppose that the quantifiable aspects of the microstructure of the material is denoted by $\Gamma$ which contains information such as dislocation density, size, distribution 
and orientation of grains in poly-crystals, etc. We consider the strain energy function of the material to be dependent on $\Gamma$ i.e., $W(\mathbf{E}, \Gamma)$ in the following form which is analogous to $\operatorname{Eqn}(7)$

$$
W(\mathbf{E}, \Gamma)=\frac{1}{2} \lambda(\operatorname{tr}(\mathbf{E}))^{2}+\mu t r\left(\mathbf{E}^{2}\right)+\frac{1}{3} C(\Gamma)(\operatorname{tr}(\mathbf{E}))^{3}+B(\Gamma) \operatorname{tr}(\mathbf{E}) \operatorname{tr}\left(\mathbf{E}^{2}\right)+\frac{1}{3} A(\Gamma)\left(\operatorname{tr}\left(\mathbf{E}^{3}\right)\right)
$$

where the following assumptions are explicitly indicated:

1. Strain energy function is dependent on the microstructure $\Gamma$,

2. Third order elastic constants are a function of $\Gamma$,

3. Change in microstructure $(\Gamma)$ does not affect the second order elastic constants $(\lambda, \mu)$.

Note that there are situations where the above assumptions are violated; e.g., when there is macroscale damage or temperature induced phase transformations. In those cases, one may have to account for the dependence of second order elastic constants $(\lambda$, $\mu)$ or may have to employ a constitutive model different from the one in Eqn(14). In fact, it is widely recognized that linear elastic parameters $\lambda$ and $\mu$ characterize the chemical bonding in the material and that these parameters are not affected by the usual defects in the crystal lattice until continuum damage mechanics comes into play via significant microcracking or void nucleation for example. Work hardening is an excellent example. The yield strength of the alloy varies considerably due to different dislocation densities and substructures associated with different amounts of cold work, but the linear elastic parameters remain essentially unchanged. Hence, in the context of nonlinear ultrasonics where the focus is on early damage detection, it is expected that the change in linear elastic properties $(\lambda$ and $\mu)$ is much smaller when compared to the change in $A(\Gamma), B(\Gamma)$ and $C(\Gamma)$ and hence the above assumptions (1-3) are reasonable. For the case of single crystals the microstructure corresponds to that of the unit cell representative of the crystal lattice; $A(\Gamma), B(\Gamma)$ and $C(\Gamma)$ correspond to the third order elastic constants of the material. While for the case of polycrystalline metals, $\operatorname{Eqn}(14)$ does not necessarily imply that $A(\Gamma)=A, B(\Gamma)=B$ and $C(\Gamma)=C$ where $A, B$ and $C$ are third-order elastic constants pertaining to the single crystals.

From hereon the constitutive relation defined by $\operatorname{Eqn}(14)$ is applied and the explicit dependence of $A, B$ and $C$ on $\Gamma$ is not indicated for brevity. We discuss the following 
aspects of the the material behavior governed by $\operatorname{Eqn}(14)$ :

1. Tension-Compression asymmetry,

2. Shear-Normal coupling and

3. Deformation induced anisotropy.

\subsection{Tension-Compression asymmetry}

The role of tension-compression asymmetry in higher harmonic generation is investigated using the constitutive model in Eqn(14). Some simple examples are presented to demonstrate that such an asymmetry is the cause of second harmonic generation (and other even higher harmonics) in materials. After that, tension-compression asymmetry is quantified by a parameter and some plausible microstructural features responsible for the asymmetry are identified. As a simple demonstration, a uniaxial deformation example is given.

Let $\mathbf{u}=\left(\begin{array}{lll}u_{1}\left(X_{1}\right) & 0 & 0\end{array}\right)$ denote the displacement field in the material. The corresponding Lagrangian strain is

$$
\mathbf{E}=\left[\begin{array}{ccc}
\frac{\partial u_{1}}{\partial X_{1}}+\frac{1}{2}\left(\frac{\partial u_{1}}{\partial X_{1}}\right)^{2} & 0 & 0 \\
0 & 0 & 0 \\
0 & 0 & 0
\end{array}\right]
$$

Using Eqns $(9,12,13 \& 15)$ and letting $\epsilon=\frac{\partial u_{1}}{\partial X_{1}}$, we get

$$
\begin{aligned}
T_{R R}(\mathbf{E})_{11} & =(\lambda+2 \mu)\left(\epsilon+\frac{1}{2} \epsilon^{2}\right)+(A+3 B+C) \epsilon^{2} \\
T_{R R}\left(\mathbf{E}^{l}\right)_{11} & =(\lambda+2 \mu)(\epsilon)+(A+3 B+C) \epsilon^{2} \\
\left(T_{R R}^{l}(\mathbf{E})\right)_{11} & =(\lambda+2 \mu)\left(\epsilon+\frac{1}{2} \epsilon^{2}\right) .
\end{aligned}
$$

The asymmetric behavior is investigated as a function of $\epsilon$ with the elastic constants provided in Table 1. While the asymmetry is not readily apparent in stress-strain curves (Liu et al., 2013a), it is indeed present as indicated by the values of stresses obtained from Eqn(16) for strains $\epsilon= \pm 0.001^{1}$ given in Table 2. Note that the material nonlinearity dominates the

\footnotetext{
${ }^{1}$ These values are for demonstration only, ultrasonic strains are significantly less.
} 
geometric nonlinearity. Even this small asymmetry can result in higher harmonic generation as shown by Chillara and Lissenden (2014).

Thus, it is established that tension-compression behavior depicted by the strain energy function in Eqn (14) is asymmetric. We will now prove that the periodic motion of a material particle in a field with asymmetric potential is asymmetric and hence generates even harmonics. Asymmetry in the motion of a particle means that the displacement-time plot of the particle is asymmetric about the equilibrium position. One such example has already been shown in Figure 1. Some simple numerical examples pertaining to a springmass system where the potential energy of the spring is asymmetric (like that of Eqn(14)) are chosen to show that the displacements of the mass indeed have higher harmonic components.

Consider the periodic motion of a particle of mass ' $m$ ' in a conservative force field defined by the potential energy function $\mathrm{U}(u) \geq 0$ where ' $u$ ' denotes the displacement. Its kinetic energy is given by $T(u)=\frac{1}{2} m \dot{u}^{2}$ where the dot denotes the time-derivative. Suppose that the initial conditions on particle displacement and velocity give a total energy $\xi$ which is constant throughout the motion i.e.,

$$
T(u(t))+U(u(t))=\xi
$$

where $u(t)$ denotes the explicit dependence of displacement on time. We now prove that if $U(u)$ is asymmetric then so is $u(t)$.

The asymmetry of $U$ is defined by $U(a) \neq U(-a), \forall a \in[-b, b]$ for some $b \in \mathbf{R}$. Also observe that since $u(t)$ is periodic so is $\dot{u}(t)$.

Proof:

Suppose $U(u(t))$ is asymmetric but $u(t)$ is symmetric. Then, for every $t_{1}>0, \exists t_{2}$ such that

$$
u\left(t_{1}\right)=-u\left(t_{2}\right) ;\left|\dot{u}\left(t_{1}\right)\right|=\left|\dot{u}\left(t_{2}\right)\right| \text {. }
$$

Since $U\left(u\left(t_{1}\right)\right)+T\left(\dot{u}\left(t_{1}\right)\right)=U\left(u\left(t_{2}\right)\right)+T\left(\dot{u}\left(t_{2}\right)\right)$ because the energy must be conserved, we have $U\left(u\left(t_{1}\right)\right)=U\left(u\left(t_{2}\right)\right)$, which is a contradiction to the assumption that $U$ is asymmetric. Thus, $u(t)$ is asymmetric.

A similar proof can be given to show that if $u(t)$ is asymmetric then so is $U(u)$. Here 
we have proved that the displacement field is asymmetric if and only if the potential is asymmetric. Since the motion of material particles during wave propagation is analogous to the periodic motion of a particle with the potential energy function replaced by the strain energy function the above ideas can be appropriately extended.

Consider now a simple example of the motion of a particle attached to a bilinear spring to depict the harmonic generation. The schematic and parameters of the spring mass system are shown in Figure 4. Four cases are depicted where the stiffness of the spring in tension is fixed and that in compression is varied. The plots of displacement with time and the corresponding FFT's are shown in Figure 5. The FFT's clearly show the zero-frequency and small second-harmonic components for the cases where the compressive stiffness is different than the tensile stiffness. Although not apparent, there are also odd-harmonic components and other higher even-harmonic components in the FFT which are more clearly evident for a small $(1 \mathrm{~N} / \mathrm{m})$ compressive stiffness as shown in Figure 6 (FFT shown on a log-scale). The generation of odd harmonic components is due to the discontinuity in stiffness at zero displacement. To better elucidate this, an elasto-plastic spring-mass system whose response is given by Eqn(19) is chosen. The parameters used for simulation are $k=10 \mathrm{~N} / \mathrm{m}, m=10 \mathrm{~kg}$ and $u_{e}=0.1 \mathrm{~m}$ and the amplitude of the inital displacement of the particle is $0.2 \mathrm{~m}$. Figure 7 shows the time domain and frequency domain responses. The FFT given in log-scale shows only the presence of odd-harmonic components. This confirms that discontinuity in stiffness is responsible for odd-harmonic generation.

$$
F=\left\{\begin{array}{cc}
-k u & \text { if }|u|<u_{e} \\
-k u_{e} \frac{u}{|u|} & \text { if }|u| \geq u_{e}
\end{array}\right.
$$

From the above study, it can be concluded that the tension-compression asymmetry in the material behavior is a cause of (even) higher harmonic generation. Since the motion of each material particle is periodic with the energy equal to that carried by the propagating wave (neglecting damping), it can be concluded that the even harmonic generation in the material described by Eqn(14) is due to asymmetry in the strain energy function. This simple case of a bilinear spring-mass system illustrates the nature of higher harmonic generation due to asymmetry. It is interesting to observe that the degree of asymmetry is proportional to the 
content of higher harmonics and also the zero frequency component as shown in Figure 5. Hence, it appears that the degree of asymmetry in the constitutive response of a material can be quantified by an energy based measure ' $\eta$ ' defined as

$$
\eta=\frac{(W(-\mathbf{E})-W(\mathbf{E}))}{(W(\mathbf{E})+W(-\mathbf{E}))}
$$

where 'E' denotes the Lagrangian strain tensor. The physical significance of the above measure becomes apparent by considering the 1D nonlinear stress-strain relation in Eqn(1). The corresponding strain energy function can be written as

$$
W(\epsilon)=\frac{1}{2} E \epsilon^{2}+\frac{1}{3} E \beta \epsilon^{3}
$$

From Eqns(20 \& 21), we have $\eta=-\frac{2}{3} \beta \epsilon$. Thus, $\eta$ can be thought of as an alternative measure of nonlinearity proportional to $\beta$. In fact, ' $\eta$ ' corresponds to the fraction of energy transferred from the primary wave to (even) higher harmonics during wave propagation. As opposed to the $\beta$ parameter defined only for the uniaxial case, ' $\eta$ ' can be applied for multiaxial stress/strain states that occur during wave propagation. Also, note that the above definition does not rely on any specific form of the strain energy function $W(\mathbf{E})$ and is applicable in a general context. Hence, the above definition (based on energy) can be employed in developing homogenization based approaches to quantify acoustic nonlinearity from various kinds of microstructures comprising dislocations, precipitates, micro-cracks, voids, etc.

Thus far, the tension-compression asymmetry has been identified as a necessary and sufficient feature of material behavior that results in even harmonic generation. In other words, any constitutive model that incorporates tension-compression asymmetry predicts even harmonic generation in the material. Below, we identify some plausible microstructural features responsible for tension-compression asymmetry.

As already noted, it can be shown that the asymmetry arises out of the terms involving $A(\Gamma), B(\Gamma)$ and $C(\Gamma)$ in Eqn(14). While Table 2 demonstrates the effect of just the higher order elastic constants, i.e., elastic nonlinearity in a single crystal, several other microstructural features may contribute to the nonlinearity especially in the scenarios where $\Gamma$ is continuously changing as in fatigue, creep or thermal aging. Residual stress is also 
an important factor that contributes to the asymmetry in the material response. When a material contains residual stress, the mean position of the oscillation of the material particle shifts to a different point on the stress-strain curve. Here, it is obvious to see that there is an asymmetry depending on the nature of the residual stress. Also, the residual stress affects the instantaneous stiffness of the material as will be illustrated in section 3.3 on deformation induced anisotropy. It is interesting to note that contact nonlinearity (Nazarov and Sutin, 1997) induced even harmonic generation can be modeled as asymmetry stemming from crack opening and closure corresponding to tension and compression phases respectively.

\subsection{Shear-normal coupling}

Shear-normal coupling is another important aspect of the material behavior, but it received less attention as most studies use 1D models that cannot capture it. Shear-normal coupling means that a simple shear deformation (Ogden, 1997) can only occur by applying normal stress in addition to the shear stress. This phenomenon does not occur in linear elasticity of isotropic materials. It occurs only in crystals having symmetry above orthotropic. Here, we demonstrate the shear-normal coupling exhibited by the strain energy function (Eqn14). Consider antiplane shear deformation $(\operatorname{Eqn}(22))$ of which simple shear is a subcase.

$$
\mathbf{u}=\left(\begin{array}{c}
0 \\
0 \\
u_{3}\left(\mathrm{X}_{1}, \mathrm{X}_{2}\right)
\end{array}\right), \mathbf{H}=\left[\begin{array}{ccc}
0 & 0 & 0 \\
0 & 0 & 0 \\
\frac{\partial u_{3}}{\partial X_{1}} & \frac{\partial u_{3}}{\partial X_{2}} & 0
\end{array}\right]
$$

Hence, the Lagrangian strain is

$$
\mathbf{E}=\frac{1}{2}\left[\begin{array}{ccc}
\left(\frac{\partial u_{3}}{\partial X_{1}}\right)^{2} & \left(\frac{\partial u_{3}}{\partial X_{1}}\right)\left(\frac{\partial u_{3}}{\partial X_{2}}\right) & \frac{\partial u_{3}}{\partial X_{1}} \\
\left(\frac{\partial u_{3}}{\partial X_{1}}\right)\left(\frac{\partial u_{3}}{\partial X_{2}}\right) & \left(\frac{\partial u_{3}}{\partial X_{2}}\right)^{2} & \frac{\partial u_{3}}{\partial X_{2}} \\
\frac{\partial u_{3}}{\partial X_{1}} & \frac{\partial u_{3}}{\partial X_{2}} & 0
\end{array}\right]
$$

To keep the mathematical expressions simple, we illustrate the shear-normal coupling using $\mathbf{T}_{R R}^{l}(\mathbf{E})$, but $\mathbf{T}_{\mathbf{R} \mathbf{R}}\left(\mathbf{E}^{l}\right)$ and $\mathbf{T}_{\mathbf{R R}}(\mathbf{E})$ are similar, just having more coupling terms. 
Using Eqns(13, 23) we get,

$$
\mathbf{T}_{R R}^{l}(\mathbf{E})=\left[\begin{array}{ccc}
(\lambda+\mu)\left(\frac{\partial u_{3}}{\partial X_{1}}\right)^{2}+\lambda\left(\frac{\partial u_{3}}{\partial X_{2}}\right)^{2} & \mu\left(\frac{\partial u_{3}}{\partial X_{1}}\right)^{2}\left(\frac{\partial u_{3}}{\partial X_{2}}\right) & \mu \frac{\partial u_{3}}{\partial X_{1}} \\
\mu\left(\frac{\partial u_{3}}{\partial X_{1}}\right)\left(\frac{\partial u_{3}}{\partial X_{2}}\right) & (\lambda+\mu)\left(\frac{\partial u_{3}}{\partial X_{1}}\right)^{2}+\lambda\left(\frac{\partial u_{3}}{\partial X_{2}}\right)^{2} & \mu \frac{\partial u_{3}}{\partial X_{2}} \\
\mu \frac{\partial u_{3}}{\partial X_{1}} & \mu \frac{\partial u_{3}}{\partial X_{2}} & \lambda\left(\left(\frac{\partial u_{3}}{\partial X_{1}}\right)^{2}+\left(\frac{\partial u_{3}}{\partial X_{2}}\right)^{2}\right)
\end{array}\right]
$$

from which it is easy to observe that there are nonzero terms of second order populating all of components except 13, 31, 23 and 32. These terms do not appear in linear elasticity and are responsible for the generation of secondary waves having a different polarization than the fundamental wave. This means that a pure transverse wave in the medium generates a longitudinal wave as a second harmonic (Goldberg, 1961). Similarly, it was experimentally shown (Liu et al., 2013b) that a shear-horizontal mode generates a second harmonic RayleighLamb mode in an aluminum plate. The harmonic generation observed here is quite different in that it does not arise from a distortion of the pulse, but rather due to the material nonlinearity and finite amplitude of the fundamental wave propagating in the material.

As mentioned earlier, crystalline anisotropy contributes to the shear-normal coupling if the crystal has symmetry above orthotropic. Considering the fact that individual grains are anisotropic, in some cases, one can expect grains to exhibit some degree of shear-normal coupling. Also, the presence of residual stresses can lead to deformation induced anisotropy (as discussed in the next section), which may also lead to shear-normal coupling. Furthermore, the motion of dislocations under the influence of the external stress may lead to the shear normal coupling as expressed by the Peach-Koehler equations (Weertman and Weertman, 1992).

\subsection{Deformation induced anisotropy}

Deformation induced anisotropy refers to the apparent anisotropy in the behavior of a material due to the load acting on it. Also, in some cases like fatigue, such anisotropy may indeed appear in the crystal lattice of grains due to the accumulated plastic strain associated with the dislocation substructures. These lead to substructural features like persistent slip bands(PSB)(Cantrell, 2004). The development of the PSB's render the response of the grain 
anisotropic due to the preferred PSB orientation. Here, we discuss how a static load renders the behavior of the material anisotropic. The propagation of ultrasonic waves in a prestressed material is analogous to the problem of small deformation superposed on a large deformation. At the meso-scale, the static load can be associated with residual stress/strain induced in the material during the manufacturing process. Cantrell (2004) assumed that the uniaxial stress state renders an initially isotropic material to have a transversely isotropic symmetry. While this assumption suffices for longitudinal wave propagation in the presence of a uniaxial stress, it should be noted that at the meso-scale this might not be true due to the inherent anisotropy of the crystalline grain. Some important aspects of wave propagation like shear normal coupling may be overlooked due to such simplifying assumptions. Deformation induced anisotropy exhibited by material governed by the strain energy function in Eqn(14) can be analyzed as follows.

Let a triaxial stretch be given by

$$
x_{1}=p_{1} X_{1} ; x_{2}=p_{2} X_{2} ; x_{3}=p_{3} X_{3}
$$

where $p_{1}, p_{2}$ and $p_{3}$ are the stretches in the three coordinate directions. The corresponding displacement gradient is given by

$$
\mathbf{H}=\left[\begin{array}{ccc}
p_{1}-1 & 0 & 0 \\
0 & p_{2}-1 & 0 \\
0 & 0 & p_{3}-1
\end{array}\right]
$$

The effective/incremental modulii (Ogden, 1997) for the material can be obtained for this deformed state. Using Eqns (6-10) and index notation, the incremental modulii for the above deformation $(\operatorname{Eqn}(25))$ are given by

$$
\begin{aligned}
\frac{\partial S_{i j}}{\partial H_{k l}}= & \lambda \delta_{i j} \delta_{k l}+\left(\mu+B H_{p p}\right)\left(\delta_{i k} \delta_{j l}+\delta_{i l} \delta_{j k}\right)+\lambda\left(H_{i j} \delta_{k l}+H_{k l} \delta_{i j}\right) \\
& +\lambda H_{p p} \delta_{i k} \delta_{j l}+\mu\left(H_{l j}+H_{j l}\right) \delta_{i k}+\mu\left(H_{i k}+H_{k i}\right) \delta_{j l}+\mu\left(H_{i l} \delta_{j k}+H_{k j} \delta_{i l}\right) \\
& +2 C H_{p p} \delta_{i j} \delta_{k l}+B\left(H_{l k}+H_{k l}\right) \delta_{i j}+B\left(H_{i j}+H_{j i}\right) \delta_{k l}
\end{aligned}
$$




$$
+\frac{A}{4}\left(\left(H_{i k}+H_{k i}\right) \delta_{j l}+\left(H_{j l}+H_{l j}\right) \delta_{i k}+\left(H_{j k}+H_{k j}\right) \delta_{l i}+\left(H_{i l}+H_{l i}\right) \delta_{j k}\right)
$$

where ' $\delta$ ' denotes the Kronecker-delta. The incremental modulii evaluated using Eqn(27) are given for two examples of stretch. In Voigt notation, the elastic constants for linear clastic material (obtained from the quadratic part of $\mathrm{W}$ in Eqn(14)) are

$$
C_{\text {linear }}=\left[\begin{array}{cccccc}
110 & 60 & 60 & 0 & 0 & 0 \\
60 & 110 & 60 & 0 & 0 & 0 \\
60 & 60 & 110 & 0 & 0 & 0 \\
0 & 0 & 0 & 25 & 0 & 0 \\
0 & 0 & 0 & 0 & 25 & 0 \\
0 & 0 & 0 & 0 & 0 & 25
\end{array}\right] \mathrm{GPa}
$$

given $\lambda$ and $\mu$ from Table 1 .

For uniaxial stretch $\left(p_{1}=1.002 ; p_{2}=1 ; p_{3}=1\right)$, the incremental modulii from Eqn $(27)$ are

$$
C_{\text {incremental }}=\left[\begin{array}{cccccc}
105.42 & 58.56 & 58.56 & 0 & 0 & 0 \\
58.56 & 107.76 & 59.24 & 0 & 0 & 0 \\
58.56 & 59.24 & 107.76 & 0 & 0 & 0 \\
0 & 0 & 0 & 24.32 & 0 & 0 \\
0 & 0 & 0 & 0 & 24.1 & 0 \\
0 & 0 & 0 & 0 & 0 & 24.1
\end{array}\right] \mathrm{GPa}
$$

which corresponds to a transversely isotropic material.

For biaxial stretch $\left(p_{1}=1.002 ; p_{2}=1.001 ; p_{3}=1\right)$, the incremental modulii are

$$
C_{\text {incremental }}=\left[\begin{array}{cccccc}
104.3 & 57.84 & 58.18 & 0 & 0 & 0 \\
57.84 & 105.47 & 58.52 & 0 & 0 & 0 \\
58.18 & 58.52 & 106.64 & 0 & 0 & 0 \\
0 & 0 & 0 & 23.87 & 0 & 0 \\
0 & 0 & 0 & 0 & 23.76 & 0 \\
0 & 0 & 0 & 0 & 0 & 23.65
\end{array}\right] \mathrm{GPa}
$$


which corresponds to an orthotropic material.

Note that the degree of anisotropy exhibited due to the load is small, but may be sufficient to create higher order effects such as higher harmonic generation. Also, the results presented above are for the case of an isotropic material subjected to deformation. In cases where the material is anisotropic to begin with, it can be expected that the induced anisotropy may correspond to higher order of symmetry and hence may contribute to shear normal coupling (discussed in the previous section). In this case the analysis follows along the same lines just presented except that the starting point would be an anisotropic elastic strain energy function which involves more constants than the one in Eqn(14). Another point to note here is that the results presented above correspond to a homogeneous initial deformation and the results may be quite different if the deformation is inhomogeneous.

Until now we described the anisotropy in the material behavior due to the presence of the external load. To describe the effect of load on harmonic generation we start with the relation $(\operatorname{Eqn}(28))$ between the first Piola-Kirchhoff stress and components of the displacement gradient for a general anisotropic material as in Cantrell (2004)

$$
S_{i j}=A_{i j k l} H_{k l}+A_{i j k l m n} H_{k l} H_{m n}+A_{i j k l m n p q} H_{k l} H_{m n} H_{p q}
$$

where, $A_{i j k l}, A_{i j k l m n}$ and $A_{i j k l m n p q}$ are the first, second and third order Huang coefficients. Suppose the pre-stress/strain generates a displacement gradient $\mathbf{H}^{\mathbf{0}}$ in the material. Equation (28) can be re-written as a Taylor-series about $\mathbf{H}^{\mathbf{0}}$ in the following way

$$
\mathbf{S}=\mathbf{S}\left(\mathbf{H}_{\mathbf{0}}\right)+\left.\frac{\partial \mathbf{S}}{\partial \mathbf{H}}\right|_{\mathbf{H}^{\mathbf{0}}}\left(\mathbf{H}-\mathbf{H}^{\mathbf{0}}\right)+\left(\left.\frac{\partial^{2} \mathbf{S}}{\partial \mathbf{H}^{2}}\right|_{\mathbf{H}^{\mathbf{0}}}\left(\mathbf{H}-\mathbf{H}^{\mathbf{0}}\right)\right)\left(\mathbf{H}-\mathbf{H}^{\mathbf{0}}\right)
$$

where $\left.\frac{\partial \mathbf{S}}{\partial \mathbf{H}}\right|_{\mathbf{H}^{\mathbf{0}}}$ and $\left.\frac{\partial^{2} \mathbf{S}}{\partial \mathbf{H}^{2}}\right|_{\mathbf{H}^{\mathbf{0}}}$ are the first and second-order Huang coefficients referred from the deformed state corresponding to $\mathbf{H}^{0}$ and are given using index notation below.

$$
\begin{aligned}
\frac{\partial S_{i j}}{\partial H_{r s}}= & A_{i j r s}+A_{i j r s m n} H_{m n}^{0}+A_{i j k l r s} H_{k l}^{0} \\
& +A_{i j r s m n p q} H_{m n}^{0} H_{p q}^{0}+A_{i j k l r s p q} H_{k l}^{0} H_{p q}^{0}+A_{i j k l m n r s} H_{k l}^{0} H_{m n}^{0} \\
\frac{\partial^{2} S_{i j}}{\partial H_{r s} H_{t w}}= & \left(A_{i j r s t w}+A_{i j t w r s}\right)+\left(A_{i j r s t w p q}+A_{i j t w r s p q}\right) H_{p q}^{0}
\end{aligned}
$$




$$
+\left(A_{i j r s m n t w}+A_{i j t w m n r s}\right) H_{m n}^{0}+\left(A_{i j k l r s t w}+A_{i j k l t w r s}\right) H_{k l}^{0}
$$

It is easy to see that first and second order Huang coefficients (responsible for harmonic generation) depend on the initial deformation $\mathbf{H}^{0}$. While the first order coefficients describe deformation induced anisotropy due to the load, the second-order coefficients describe the effect of load on the harmonic generation.

\section{Closure}

A brief review of the theory of nonlinear ultrasound has been presented. Tensioncompression asymmetry, shear-normal coupling and deformation induced anisotropy aspects of material behavior were discussed using the constitutive model $(\operatorname{Eqn}(14))$. The above features should be interpreted at the appropriate length-scale. For example, we do not claim that the macroscopic behavior (as obtained from mechanical testing) of polycrystalline metal shows tension-compression asymmetry, but a meso-scale constitutive model should incorporate it so that it predicts (even) harmonic generation.

Tension-compression asymmetry was identified as a cause of even harmonic generation. A bilinear spring-mass system was investigated as a simple mechanical analogue to describe particle motion during the nonlinear wave propagation and it was observed that

- the degree of asymmetry was proportional to the amplitude of higher harmonic generation, and

- the discontinuity in the modulus (spring constant) was responsible for the generation of odd harmonics.

Additionally, the contributions of geometric and material non-linearities to the tensioncompression asymmetry exhibited by Eqn(14) were computed. The degree of asymmetry was quantified using an energy based measure ' $\eta$ ' in Eqn(20). Relevant microstructural features responsible for tension-compression asymmetry in metals were identified.

Shear-normal coupling was identified to be the cause of higher harmonic generation of a wave having different polarization than the primary wave. Some plausible microstructural features responsible for shear-normal coupling were identified. Likewise, deformation 
induced anisotropy due to the finite pre-stress/strain was modeled using incremental modulii computed using Eqn(27). Two cases of uniaxial and biaxial stretch demonstrated the effect. Residual stresses/strains were also identified to be sources of deformation induced anisotropy. The effect of pre-stress /strain on the harmonic generation is qualitatively described.

The aspects of material behavior discussed in this article are general in that any constitutive model which incorporates them shows similar behavior to that discussed for the elastic strain energy function Eqn(14). For example, the $\eta$ parameter defined in Eqn(20) is valid in a general context and is independent of the specific form of $W(\mathbf{E})$. The motivation behind this study was two-fold:

1. Guide the meso-scale model development by identifying necessary and sufficient features to be incorporated for studying higher harmonic generation.

2. Quantify the contribution of different microstructural features to higher harmonic generation.

The first objective has been accomplished in that we were able to identify tension-compression asymmetry as a cause for sccond (even) harmonic gencration and shear normal coupling to be the cause of generation of second harmonic longitudinal (Rayleigh-Lamb) waves from transverse (Shear-Horizontal) waves. To quantify the contribution of different microstructural features to harmonic generation, it suffices to evaluate the contribution of each microstructural feature (dislocation substructures, precipitates, micro-cracks/voids, etc) to the tension compression asymmetry and shear normal coupling. In this regard, homogenization based approaches seem to be fruitful and our preliminary investigation concerning the use of tension-compression asymmetry to quantify harmonic generation from material with micro-voids is reported in Chillara and Lissenden (2015). 


\section{References}

Baby S., Nagaraja Kowmudi B., Omprakash C. M., Satyanarayana D. V. V., Balasubramaniam K. and Kumar V., 2008, "Creep damage assessment in titanium alloy using a nonlinear ultrasonic technique", Scripta Materialia, 59(8), 818-821.

Bowen R.M., Introduction to continuum mechanics for engineers, Plenum, New York, 1989.

Breazeale M.A., Thompson D.O., 1963, "Finite-amplitude ultrasonic waves in aluminum", Applied Physics Letters, 3, 77.

Buck O., 1976, "Harmonic generation for measurement of internal stresses as produced by dislocations.", IEEE Transactions on Sonics and Ultrasonics, 23(5), 346-350.

Cantrell J.H. and Yost W.T., 2001, "Nonlinear ultrasonic characterization of fatigue microstructures", International Journal of fatigue, 23, 487-490.

Cantrell J.H., 2004, "Substructural organization, dislocation plasticity and harmonic generation in cyclically stressed wavy metals", Proceedings of Royal Society of London A, 460, 757- 780 .

Cantrell J.H., 2006, "Quantitative assessment of fatigue damage accumulation in wavy slip metals from acoustic harmonic generation", Philosophical Magazine, 86, 11, 1539-1554.

Cantrell J.H., 2009, "Nonlinear dislocation dynamics at ultrasonic frequencies", Journal of Applied Physics, 105, 043520.

Cash W.D. and Cai W., 2011, "Dislocation contribution to acoustic nonlinearity: The effect of orientation-dependent line energy", Journal of Applied Physics, 109, 014915.

Cash W.D. and Cai W., 2012, "Contribution of dislocation dipole structures to the acoustic nonlinearity", Journal of Applied Physics, 111, 074906.

Chillara V.K. and Lissenden C.J., 2014, "Nonlinear guided waves in plates: A numerical perspective", Ultrasonics, 54, 6, 1553-1558. 
Chillara V.K. and Lissenden C.J., 2015, "Towards a micro-mechanics based understanding of ultrasonic higher harmonic generation", Proc. SPIE 9438, Health Monitoring of Structural and Biological Systems 2015, 94380R (March 23, 2015).

Destrade M. and Ogden R. W., 2010, "On the third-and fourth-order constants of incompressible isotropic elasticity", Journal of the Acoustical Society of America, 128, 3334.

Goldberg Z. A., 1961, "Interaction of plane longitudinal and transverse elastic waves", Sov. Phys. Acoust, 6, 306-310.

Hikata A., Chick B.B., Elbaum C., 1965, "Dislocation contribution to the second harmonic generation of ultrasonic waves", Journal of Applicd Physics, 36, 229.

Hikata A. and Elbaum C., 1966a, "Generation of ultrasonic second and third harmonics due to dislocations 1", Physical Review, 144, 2, 469-477.

Hikata A. and Elbaum C., 1966b, "Generation of ultrasonic second and third harmonics due to dislocations 2", Physical Review, 151, 2, 442-449.

Jhang K. Y., 2009, "Nonlinear ultrasonic techniques for nondestructive assessment of micro damage in material: a review", International journal of precision engineering and manufacturing, 10(1), 123-135.

Kim C. S. and Lissenden, C. J., 2009, "Precipitate contribution to the acoustic nonlinearity in nickel-based superalloy", Chinese Physics Letters, 26(8), 086107.

Landau.L.D. and Lifshitz E.M., 1970, Theory of Elasticity, $2^{\text {nd }}$ ed., Permagon Press, New York.

Liu Y., Lissenden C. J. and Rose J. L., 2013a, "Cumulative second harmonics in weakly nonlinear plates and shells", In SPIE Smart Structures and Materials+ Nondestructive Evaluation and Health Monitoring, 86950S-86950S.

Liu Y, Chillara V.K. and Lissenden C.J., 2013b, "On selection of primary modes for generation of strong internally resonant second harmonics in plates", Journal of Sound and Vibration, 33, 19, 4517-4528. 
Matlack K. H., Wall J. J., Kim J. Y., Qu J., Jacobs L. J. and Viehrig H. W., 2012, "Evaluation of radiation damage using nonlinear ultrasound", Journal of Applied Physics, 111(5), 054911.

Nazarov V. E. and Sutin A. M., 1997, "Nonlinear elastic constants of solids with cracks", The Journal of the Acoustical Society of America, 102(6), 3349-3354.

Ogden R.W., 1997, Non-linear elastic deformations, Dover Publications, Mineola, New York.

Valluri J. S., Balasubramaniam K and Prakash R. V., 2010, "Creep damage characterization using non-linear ultrasonic techniques", Acta Materialia, 58(6), 2079-2090.

Van Den Abeele KE-A., Johnson P.A. and Sutin A, 2000a, "Nonlinear elastic wave spectroscopy (NEWS) techniques to discern material damage, part I: nonlinear wave modulation spectroscopy (NWMS)", Research in nondestructive evaluation, 12(1), 17-30.

Van Den Abeele KE-A., Johnson P.A. and Sutin A., 2000b, "Nonlinear elastic wave spectroscopy (NEWS) techniques to discern material damage, Part II: Single-mode nonlinear resonance acoustic spectroscopy", Research in Nondestructive Evaluation, 12(1), 31-42.

Weertman J. and Weertman J.R., 1992, Elementary Dislocation Theory, Oxford University press, New York.

Xiang Y., Deng M. and Xuan F.Z., 2014, "Creep damage characterization using nonlinear ultrasonic guided wave method: A mesoscale model.", Journal of Applied Physics $115(4), 044914$.

Zheng Y., Maev R. G. and Solodov I. Y., 2000, "Review/Sythse Nonlinear acoustic applications for material characterization: A review", Canadian Journal of Physics, 77(12), 927-967. 


\section{Captions}

Table 1 Elastic constants (in GPa) used for the study

Table 2 Contribution of material and geometric nonlinearities to the asymmetery in stress $(\mathrm{MPa})$

Figure 1 Asymmetric sine wave generates even harmonics

Figure 2 Triangular wave generates odd harmonics

Figure 3 Trapezoidal wave generates odd harmonics

Figure 4 Schematic showing the parameters of the spring mass system

Figure 5 Displacement vs time for different spring stiffnesses in compression, and their corresponding FFT's

Figure 6 Displacement vs time for compression spring stiffness $=1 \mathrm{~N} / \mathrm{m}$ and its corresponding FFT on a log-scale

Figure 7 Displacement vs time for the elasto-plastic spring-mass system and its corresponding FFT on a log-scale 
Table 1: Elastic constants (in GPa)

\begin{tabular}{ccccc}
\hline \hline$\lambda$ & $\mu$ & $A$ & $B$ & $C$ \\
\hline 60 & 25 & -320 & -200 & -190
\end{tabular}

Table 2: Contribution of material and geometric nonlinearities to the asymmetery in stress( $\mathrm{MPa})$

\begin{tabular}{cccc} 
& $T_{R R}(\mathbf{E})_{11}($ Eqn 9$)$ & $T_{R R}\left(\mathbf{E}^{l}\right)_{11}($ Eqn 12) & $\left(T_{R R}^{l}(\mathbf{E})\right)_{11}($ Eqn 13) \\
\hline \hline Strain $(\epsilon)$ & Total (Material+Geometric) & (Material nonlinearity) & (Geometric nonlinearity) \\
\hline 0.001 & 108.95 & 108.89 & 110.06 \\
-0.001 & -111.05 & -111.11 & -109.94
\end{tabular}



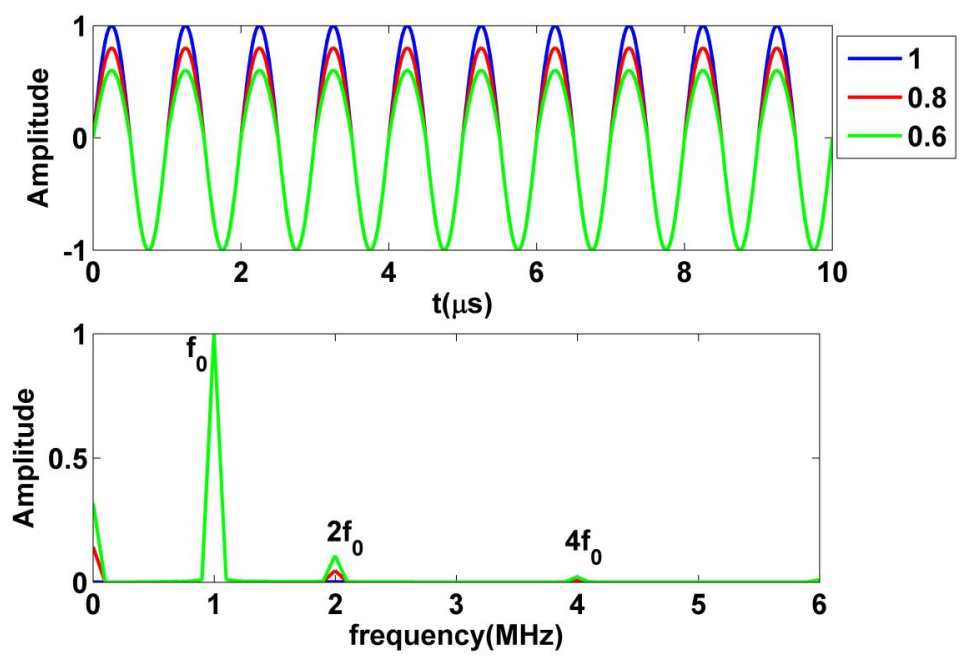

Figure 1: Asymmetric sine wave generates even harmonics
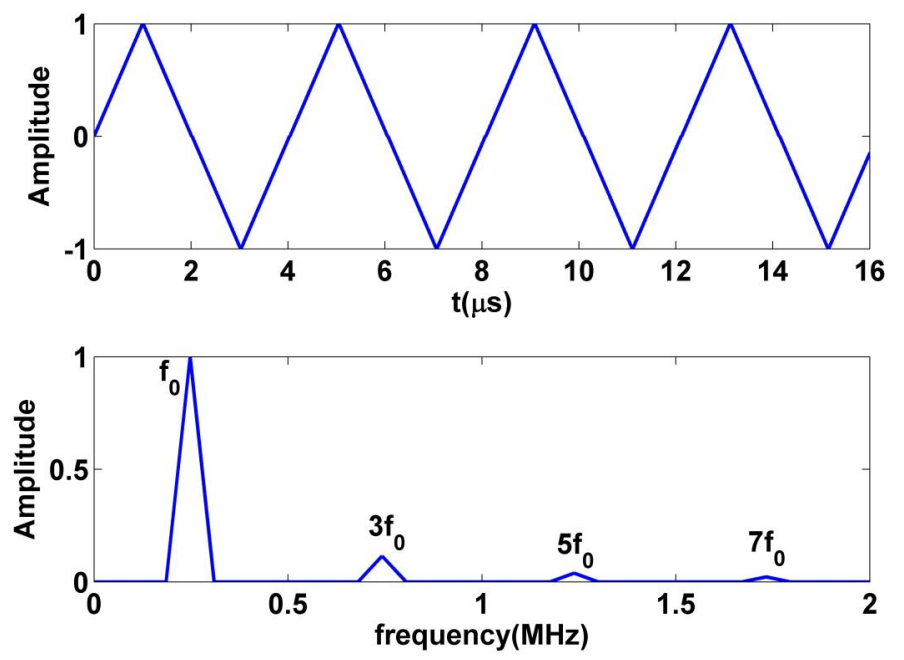

Figure 2: Triangular wave generates odd harmonics 

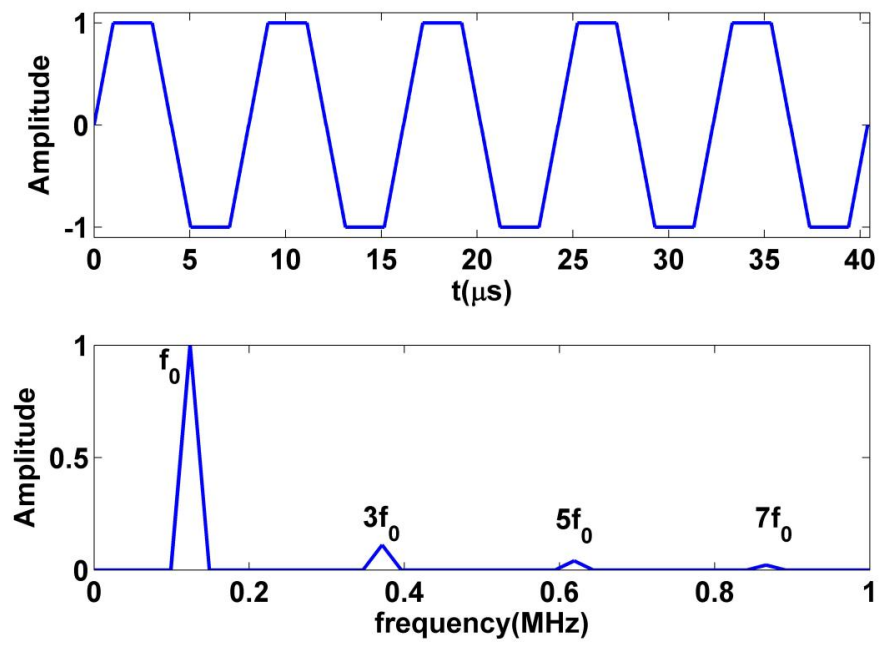

Figure 3: Trapezoidal wave generates odd harmonics

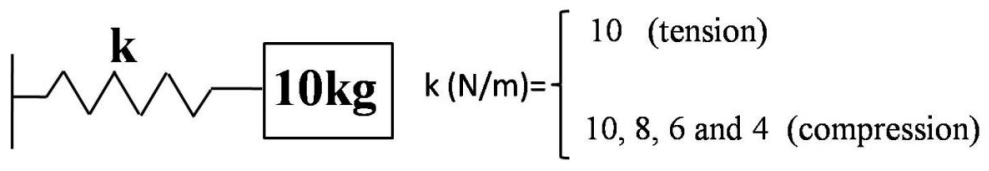

Figure 4: Schematic showing the parameters of the spring mass system
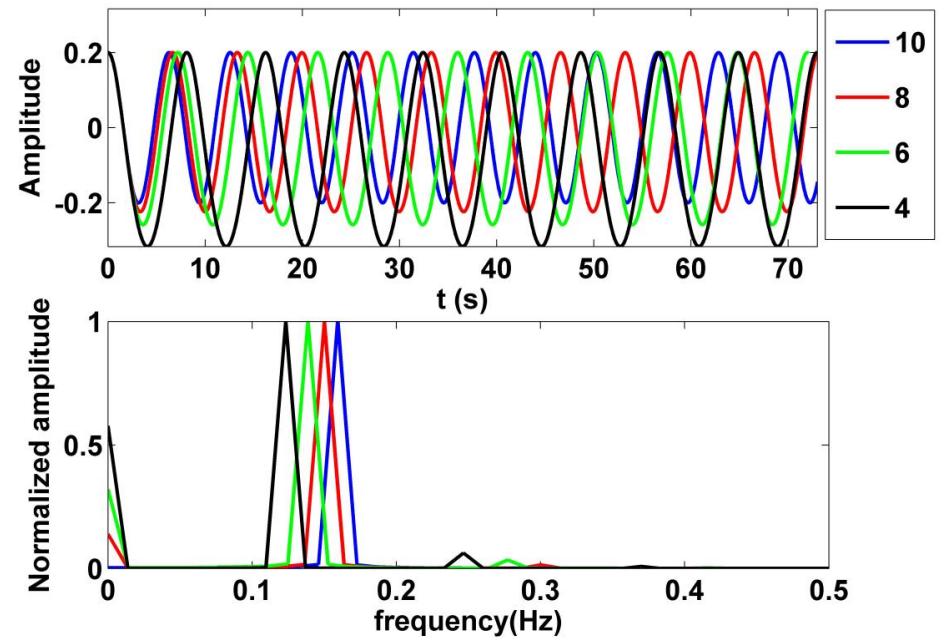

Figure 5: Displacement vs time for different spring stiffnesses in compression (spring stifness in tension=10 $\mathrm{N} / \mathrm{m}$ ), and their corresponding FFT's 

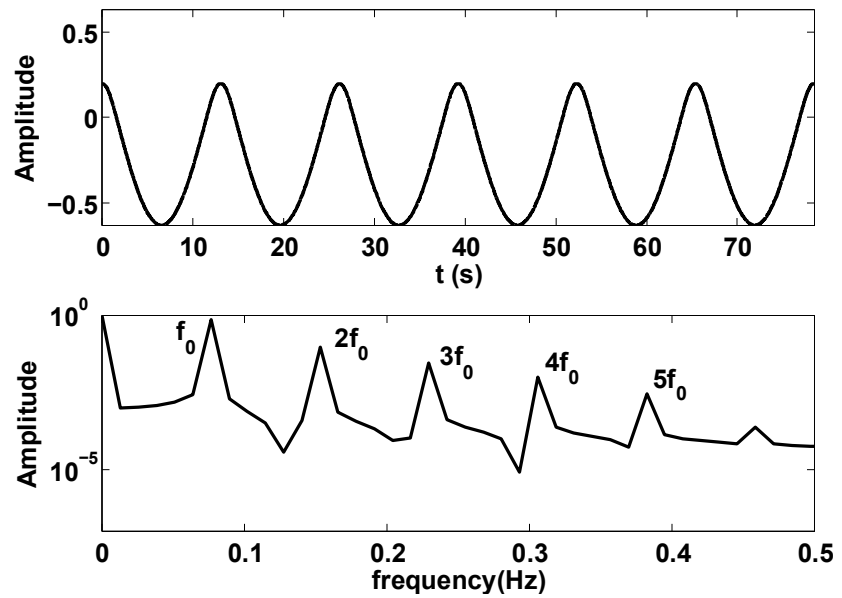

Figure 6: Displacement vs time for compression spring stiffness $=1 \mathrm{~N} / \mathrm{m}$ (stiffness in tension=10 N/m) and its corresponding FFT on a log-scale 

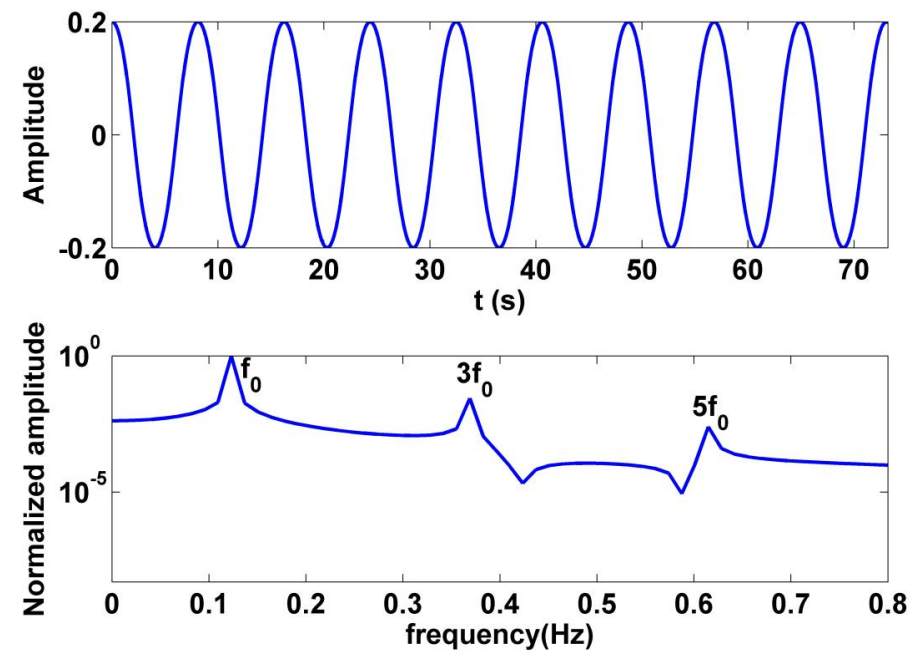

Figure 7: Displacement vs time for the elasto-plastic spring-mass system and its corresponding FFT on a log-scale 